\title{
Editorial
}

\section{Toxicity of Environmental Contaminants}

\author{
Sunil Kumar, ${ }^{1}$ Patrick Hettiaratchi, ${ }^{2}$ Nanjappa Ashwath, ${ }^{3}$ and Petros Gikas ${ }^{4}$ \\ ${ }^{1}$ Solid and Hazardous Waste Management Division, CSIR-NEERI, Nehru Marg, Nagpur 440 020, India \\ ${ }^{2}$ Department of Civil Engineering, Center for Environmental Engineering Research and Education, University of Calgary, \\ Calgary, AB, Canada T2N 1N4 \\ ${ }^{3}$ Centre for Plant and Water Science, Central Queensland University, Rockhampton, QLD 4702, Australia \\ ${ }^{4}$ Department of Environmental Engineering, Technical University of Crete, 73100 Chania, Greece \\ Correspondence should be addressed to Sunil Kumar; sunil_neeri@yahoo.co.in
}

Received 22 June 2015; Accepted 22 June 2015

Copyright (C) 2015 Sunil Kumar et al. This is an open access article distributed under the Creative Commons Attribution License, which permits unrestricted use, distribution, and reproduction in any medium, provided the original work is properly cited.

Environmental toxicology is one of the most interdisciplinary subjects and it aims to study the effects of toxic chemicals on living organisms using various toxicological methods. The experiments that examine the deleterious effects of environmental pollutants on animals provide scientific basis for developing environmental standards. Results of such experiments and the techniques and practices used to minimize environmental effects of toxic chemicals will help in formulating sound environmental policies and appropriate decision-making.

The special issue addresses various topics, such as biomarkers for environmental pollution monitoring; bioaccumulation of chemicals; bioassays and biomarkers in water monitoring and biomonitoring of water bodies; ecotoxicity of emerging chemicals; biological effects of monitoring; laboratory techniques and field quantitative structure-activity relationships (QSAR); ecosystem health and environmental health risk assessment.

This special issue is a compilation of selected peer reviewed papers that range from biomarkers, bioconcentration and toxication, oxidative stress defuse pathways to cytotoxic effects of pesticides, phytoremediation of polluted soil, and so forth.

The editors believe that the results presented in these papers are valuable and will stimulate discussion on further development of potential techniques and devices for effective assessment of deleterious effects of environmental pollutants on animals and the environment.

\section{Acknowledgments}

The editors thank the authors for their contribution to the new knowledge. They are also grateful to the reviewers who have spared their productive time for assessing the papers.

Sunil Kumar

Patrick Hettiaratchi Nanjappa Ashwath

Petros Gikas 

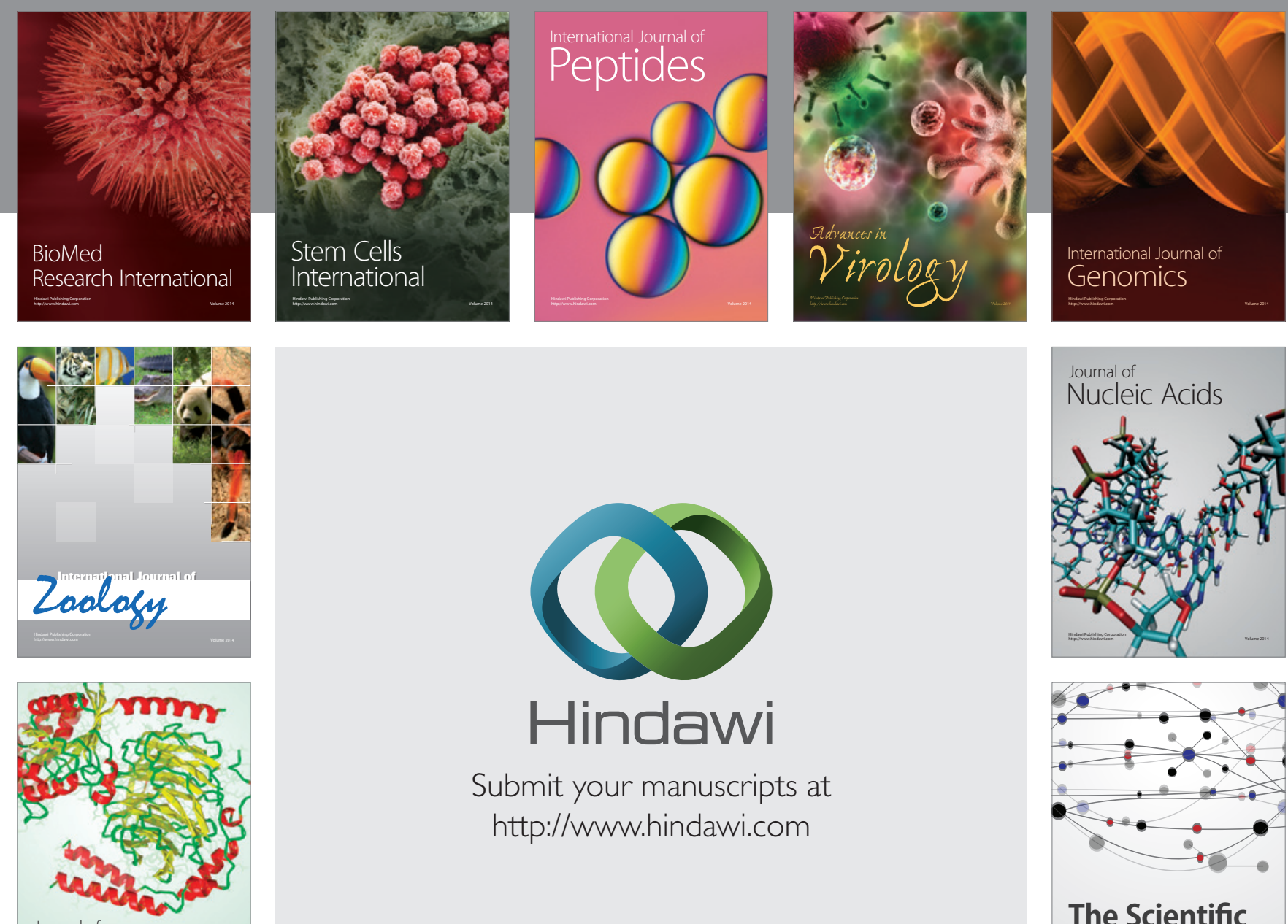

Submit your manuscripts at

http://www.hindawi.com

Journal of
Signal Transduction
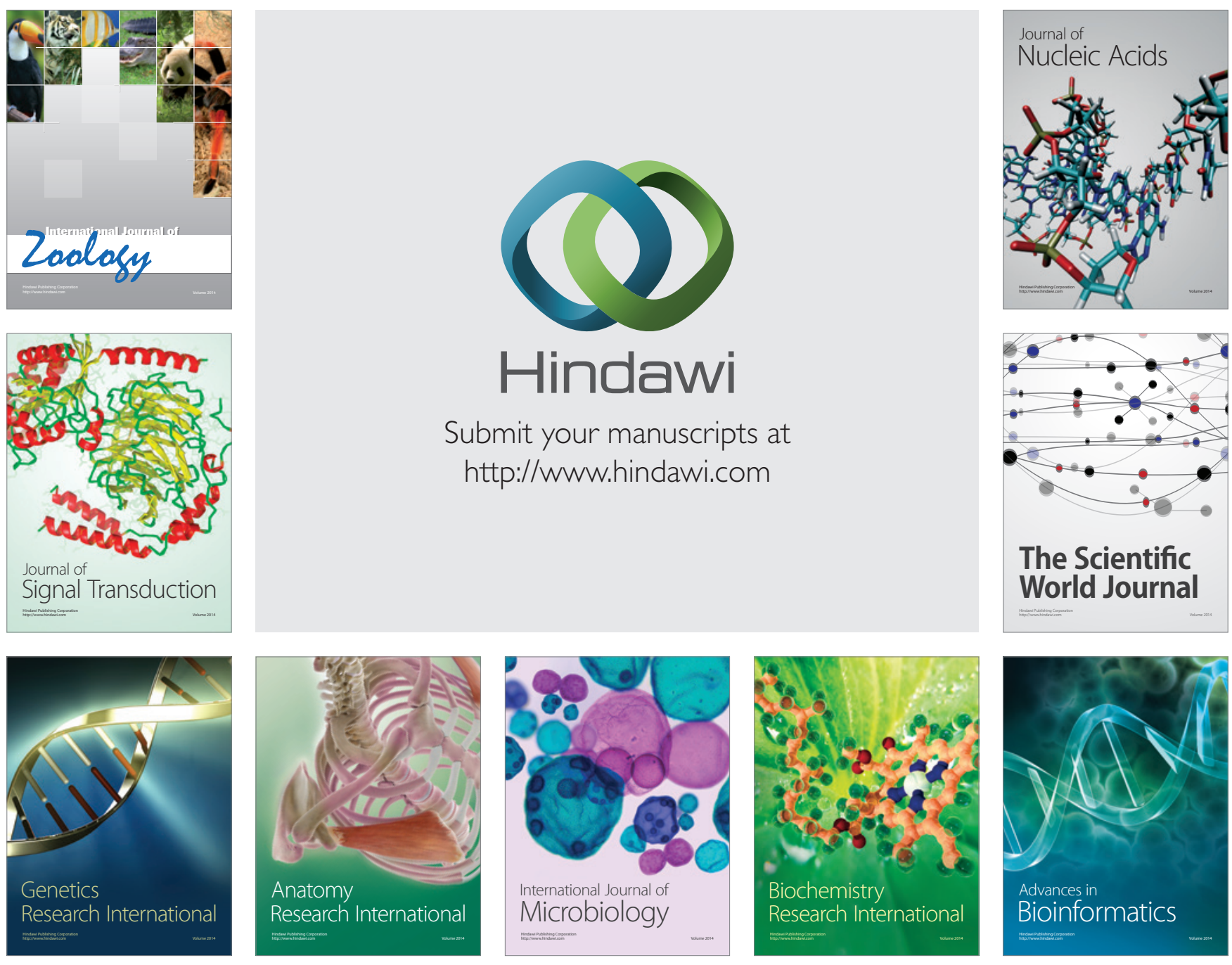

The Scientific World Journal
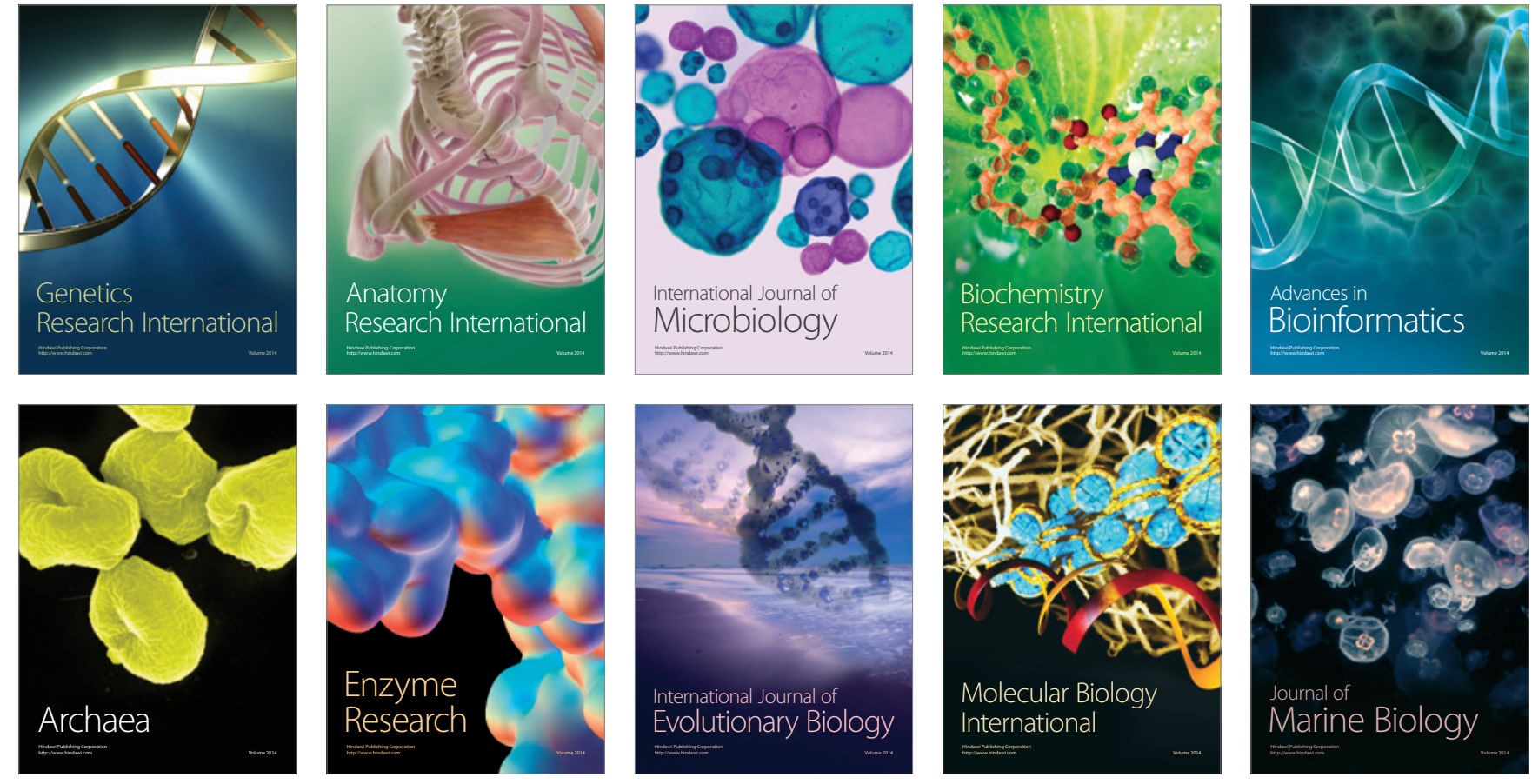